
\title{
25 Research Soure \\ Using a Discrete Choice Experiment to Examine the Factors Influencing Clinical Trial Participation in a Nationally Representative Sample
}

Michelle Queally ( $\sim$ michelle.queally@gmit.ie )

Galway-Mayo Institute of Technology - Galway Campus Library: Galway-Mayo Institute of Technology Libraries https://orcid.org/0000-0002-1259-7874

\section{Declan Devane}

National University of Ireland Galway

Matthew D. Griffin

National University of Ireland Galway

Paddy Gillespie

National University of Ireland Galway

Abhay Pandit

National University of Ireland Galway

\section{Research Article}

Keywords: Clinical trial participation, discrete choice experiment, clinical trial design

Posted Date: June 21st, 2021

DOl: https://doi.org/10.21203/rs.3.rs-498686/v1

License: (c) (1) This work is licensed under a Creative Commons Attribution 4.0 International License. Read Full License 


\section{Abstract}

Introduction

Understanding the key factors that inform an individual's decision-making process for participating in a clinical trial (CT) can improve the efficiency of recruitment for CT studies. Currently, little is known, in quantitative terms, about the relative importance of the different factors that influence CT participation decisions.

Objective

The objectives of this study were to design and conduct a discrete choice experiment (DCE) to identify critical factors, or attributes, that influence CT participation, and to estimate public preferences for, and trade-offs between, different levels of these attributes.

Methods

A DCE was designed, with input from a public patient involvement (PPI) panel and conducted via an online survey of 525 nationally representative Irish citizens. The attributes identified and included in the DCE to inform CT participation included: (1) communication, (2) financial incentive, (3) likelihood of benefit, (4) risk of side effects, and (5) follow up period. All respondents were presented with a set of choice cards and asked to choose between participating in one of two CTs, differentiated by their attributes levels, or opt-out. A series of econometric statistical regression models were estimated to generate the results of interest.

Results

Respondents had preferences which, in order of relative importance, had a lower risk of side effects, a higher likelihood of benefit, a shorter follow up, information communicated by the respondent's doctor or nurse, and a financial incentive for participation.

Conclusion

To the best of our knowledge, this is the first study to utilise a DCE to estimate preferences of the public regarding factors influencing CT participation. These findings may be useful for future CT design as they enable researchers to elicit how individuals may weigh up their choices and consider trade-offs between factors that affect their decision to participate. This study was also the first DCE to include a PPI panel to help inform the DCE design and development.

\section{Background}

A clinical trial is a research study that explores and evaluates whether a medical strategy, treatment or device is safe and effective for humans (Kerr, Knox et al. 2008). A key determinant of success for any 
clinical trial is recruiting and retaining a study population of adequate sample size (Gul and Ali 2010). Nonetheless, failure to enrol a sufficient number of study subjects in a clinical trial is a common and longstanding problem. For example, an analysis of registered clinical trials revealed that $19 \%$ were closed or terminated early because they did not recruit enough participants (Carlisle, Kimmelman et al. 2015). A recent systematic review reported that less than $50 \%$ of clinical trials meet their recruitment targets (Treweek, Pitkethly et al. 2018). It follows that if clinical trials fail to reach the recruitment target necessary for an adequately powered study and statistical analysis, significant questions arise over whether such clinical trials should be continued from an ethical standpoint, and how the clinical trial results from such studies should be interpreted and potentially used to inform healthcare decision making (Fogel 2018). Poor clinical trial recruitment practices can also cause significant financial issues, such as additional costs associated with the need for research study extensions to meet targets (Bower, Wallace et al. 2009). Thus, failures in meeting clinical trial recruitment goals have essential scientific, financial, ethical, and policy implications. Perhaps most importantly, the inability to meet clinical trial recruitment targets may exacerbate ill health impacts for patients by hindering efforts to more effectively diagnose, treat, or prevent disease. Taken together, it is incumbent for clinical trial researchers to work towards a better understanding of clinical trial participation decisions and in doing so, to improve the conduct of clinical trial research. To this end, there is a need to engage with patients and the general public to elicit their views and preferences regarding clinical design and operation.

Many studies have investigated the factors influencing participation in clinical trials from a range of different perspectives within health services research. (Walsh and Sheridan, 2016, Kurt, Semler et al., 2017). In a study examining participation in cancer clinical trials, the possibility of improving one's treatment, being keen to help others, and contributing to scientific research was identified as motivating factors (Moorcraft, Marriott et al. 2016). A narrative review identifying the factors affecting patient participation in clinical trials in Ireland reported that the primary motivating factor is obtaining a form of personal gain through participation in the trial and other factors including physician influence, cost, altruism, communication, demographics (Walsh and Sheridan 2016).

Nonetheless, much remains misunderstood. An example of a recent initiative in this literature is the PRioRiTy study (Healy, Galvin et al. 2018), which aimed to identify and prioritise crucial unanswered trial recruitment research questions. Notably, one of the critical priority research questions identified regarding clinical trial recruitment was the need for a greater understanding of the motivating factors influencing members of the public. To date, the majority of studies which examine clinical trial participation decisions have primarily been qualitative (Newton and APPIAH-POKU 2007, Sood, Prasad et al. 2009, McCann, Campbell et al. 2010, Chu, Kim et al. 2015, Walsh and Sheridan 2016). While these contributions are valuable and informative in providing a general understanding of clinical trial participation, such studies are often limited in terms of their sample size and representativeness, and the generalisability of their findings are, therefore, open to question. Furthermore, they generally do not assess the relative importance of the multiple factors that influence the individual decision to participate in a clinical trial. The latter may be an essential consideration in clinical trial design, in that researchers may often have to 
make pragmatic decisions that involve trade-offs between the set of factors which influence the clinical trial participation decision.

To advance a more comprehensive understanding of the factors that influence clinical trial participation and estimate their relative importance, this study applies a discrete choice experiment (DCE) methodological approach. A DCE is a valid, reliable and widely applied survey methodology for eliciting patient or public preferences (Bryan et al., 2000; Bryan and Parry, 2002). This methodology is an appropriate approach to gain a greater understanding of the relative importance that individuals place on the factors that influence the decision to participate in a clinical trial. Moreover, it enables the quantitative analysis of how individuals value and the trade-off between these factors when making clinical trial participation decisions. Indeed, DCEs have been identified as being superior to traditional attitudinal, satisfaction and ranking studies because they enable measurement of the strength of preferences for crucial aspects of a decision in a way that is not possible with a simple ranking of preferences, for example, such as those in qualitative methodology (Lancaster 1966, McFadden 1986). In employing this methodology in this context, this study is intended to add to the existing evidence base surrounding clinical trial participation, to offer insight regarding the trade-offs that potential clinical trial participants face in making their decision, and to provide information that may go to improve clinical trial recruitment strategies in the future.

\section{Methods}

\section{Discrete Choice Experiments}

DCEs are a widely used attribute-driven quantitative survey method which generally seeks to quantify the preferences of a sample of respondents, such as patients or the general public, relating to a particular hypothetical choice posed by the researchers (Louviere, Hensher et al. 2000, Ryan, Gerard et al. 2007). In the health context, the hypothetical choice could be a decision relating to a health outcome, healthcare service or intervention, healthcare funding mechanism, or, as in this case, a research study (Ryan 1999). Respondents are presented with a series of choices, usually between two alternatives and an opt-out option, with the former described by a set of attributes and differentiated by varying levels of the same attribute (Lancsar and Louviere 2008). By observing the respondents' selections of their preferred choices, researchers can infer and statistically estimate the values placed on different attribute levels relating to the choice set (Lancsar and Louviere 2008). For example, in this study, we employ the DCE method to infer the values that a sample of the general public place on the different factors that might influence an individual's hypothetical decision to participate in a clinical trial. In economic terms, Lancaster (1966) argued that it is the attributes of a 'good' that determines its 'utility' and, as a result, its utility can be expressed as a function of its attributes. In this study, we define the 'good' of interest as participation in a clinical trial. In conducting the DCE, we estimate a utility function for clinical trial participation derived from the preferences for individual factors that influence the clinical trial participation decision. For this study, we followed the guidance about the best approach for undertaking a DCE (Johnson, Lancsar et al. 2013). In the subsections that follow, the key methodological steps adopted are summarised. 


\section{Development and Selection of Attributes}

As an attribute-based experiment, a DCE's validity largely depends on the researchers' ability to specify attributes and their levels appropriately. In this study, we sought to ensure that the attribute development process was rigorous, systematic and transparently reported. Figure 1 below outlines the attribute development process.

\section{Fig 1. Attribute Development Pathway}

As Fig 1 shows, we initially consulted with the literature in the area of clinical trial participation (Moorcraft, Marriott et al. 2016, Kurt, Kincaid et al. 2017, Schilling, Behrens et al. 2019). Four iterative focus groups $(n=20)$ were conducted to guide the attribute and corresponding attribute level development in the next stage. These were conducted until the researcher reached theoretical saturation; that is, the point at which a range of ideas have been heard, and no new information is emerging. Adults aged 18 years and over were invited to participate in the focus group via national and local newspaper and radio advertisement. The Nominal Group Technique (NGT) (Horton 1980), a structured, multistep, facilitated group meeting technique, was used to elicit and prioritise responses to identify the most important clinical trial participation attributes. The first two focus groups were used to identify and rank attributes, the third focus group examined the corresponding attribute levels in more detail, and the fourth focus group was used to reach consensus on which attributes and levels to use in the DCE.

Also, we consulted with a public patient involvement (PPI) group to ensure that the development of the DCE questionnaire and its attribute descriptions, were as clear and easily understood as possible. In brief, members of the PPI panel helped inform a more precise description of how we explained a clinical trial and informed explanations of the attribute and level descriptions. Feedback about other design elements, including the length, question ordering, layout, specific wording, and comprehensibility, was also obtained from the panel. In particular, the panel indicated that the initial explanations presented of the survey's attribute and levels were burdensome to read and somewhat confusing. We then created a short animated clip that explained the attributes and corresponding levels in a clear, concise manner from this feedback.[1] This was a key strength and innovation of this study. Features of DCEs such as the number of attributes, levels, alternatives and choice tasks can difficult to interpret and complete for respondents. (Bech, Kjaer et al. 2011, Spinks and Mortimer 2015, Pearce, Harrison et al. 2020).

We conducted a three-stage pilot to construct the final version of the questionnaire. In the first stage, we applied the think-aloud methodology (Van Someren, Barnard et al. 1994), whereby respondents were asked to describe their thoughts about the attributes and levels identified in the experiment and the choices they were making (Ryan et al., 2009). This further examined if respondents understood the questionnaire. After that, we refined the format and fine-tuned the survey according to the feedback 
provided. Then, the refined questionnaires were tested in a pre-pilot study, in which we conducted four prepilot interviews with a convenient sample of men and women aged between 18 and 40 years of age. For the pre-pilot interviews, a think-aloud methodology was once again employed, which indicated no signs of response fatigue observed among the respondents during the completion of the survey. After a final set of minor changes to wording and a re-ordering of questions, the final pilot phase involved a pilot study with 45 respondents.

As outlined in Table 1, the final set of attributes identified through this rigorous process and included in the DCE were: (1) communication, (2) financial incentive, (3) likely benefit, (4) risk of side effects, and trial follow up. To facilitate the respondents in completing the DCE questionnaire, a short YouTube animated clip was created to explain these attributes and their levels to all survey respondents. Two of the attributes identified in this study as necessary to the participant regarding trial participation are "Likely Benefit" and Risk of Side Effect" are also key areas to consider when presenting information to potential participants in a trial. Similarly, in this DCE context, these concepts can be difficult for people to understand and accurately interpret, thus it is important to ensure that respondents understand these attributes (Harrison, Rigby et al. 2014). A literature review to establish the best approaches to describe the risk and benefit attribute found little consensus in this area. For example, a recent systematic review reported that there is as yet no exact optimal method for improving participant understanding regarding risk communication (Coyle and Gillies 2020). The review also noted that there was no clear consensus on how understanding risk affects willingness to participate in a clinical trial and called for further research around the evidence base surrounding risk communication in clinical trials. Further consultation with the PPI panel was undertaken to ensure the attributes were clearly described. The attribute description is outlined in table. As previously noted, attributes were described to respondents using an animated clip.

\section{Table 1 Attributes and Levels Descriptions}




\begin{tabular}{|ll}
\hline Attribute Levels & Attribute \\
& Explanation
\end{tabular}

Communication Information about the trial will be communicated to you by your GP or practice nurse at your primary care centre

This outlines who will communicate information about the clinical trial to

Or you.

Information about the trial will be communicated to you by the clinical trial team.

Financial

$€ 0 € 100 € 150 € 250 € 300 € 450$

Incentive

This is the amount of money that you will receive as an incentive to participate in the clinical trial.

Likely Benefit

(as a result of participating in the clinical trial)
1. No benefit: There is no chance that the healthcare approach will improve your health, but the evidence generated by the clinical trial research may improve the health of others like you in the future.

2.

3. Moderate benefit: There is a 1 in 20 chance that the specific treatment approach will lead to some improvements in your health, which may not be obvious to you and the evidence generated by the clinical trial research may improve the health of others like you in the future.

4.

5. Large benefit: There is a 1 in 20 chance that the specific treatment approach will lead to a meaningful improvement in your health, which you will notice in your day to day life and the evidence generated by the clinical trial research may improve the health of others like you in the future.
Risk of side effects
1. Minimal risk: There is a 1 in 20 chance of an adverse side effect on the specific treatment approach leading to a GP visit. The trial team will cover the cost of this.

2.

3. Moderate risk: There is a 1 in 20 chance of an adverse side effect to the specific treatment approach leading to an accident and emergency (A\&E) admission. The trial team will cover the cost of this.
This describes the likely benefit that would be gained as a result of participating in the clinical trial.
This describes the likely risk of a side effect as a result of participating in the clinical trial.

4. 
5. High risk: There is a 1 in 20 chance of an adverse side effect to the specific treatment approach leading to several nights stay as an inpatient in the hospital. The trial team will cover the cost of this.

Follow uptime Up to 1 year

Up to 4 years
This is the length of time that the trial team will be monitoring you and collecting data for the trial.

\section{Experimental Design and Final Questionnaire}

To create the multiple-choice card sets for the survey, as per methodological guidance, we used Ngene (ChoiceMetrics, version 1.1.1) to create a Bayesian efficient experimental design based on the minimisation of the Bayesian D-error (Db-error) criterion (Bliemer, Rose et al. 2008) In this case, the number of attributes and levels combinations (e.g. $2 * 2$ levels (i.e. communication; follow up); $2 * 3$ levels (i.e. likely benefit; risk of side effects); $1 * 6$ levels (i.e. financial incentive)) identified led to a vast number of choice tasks for a full-factorial design, which was thereby, deemed impractical (Johnson, Lancsar et al. 2013). The adopted technique provided a practical and pragmatic solution to this problem (Bliemer, Rose et al. 2008). In particular, the Bayesian design approach takes into account the uncertainty about the assumed prior parameter values. In brief, a prior distribution is assumed for the parameters in the design stage, which is then incorporated into the appropriate design criterion. For this DCE, priors were taken from the focus groups to inform the experimental design's initial pre-pilot construction. Choice sets for the pilot survey were developed based on priors gleaned from the pre-pilot study. After the pilot, the results were analysed, and priors were used from the pilot study to inform the experimental design's construction for the main study.

This process resulted in 36 choice sets being generated, with a blocking design of three blocks of 12 choice cards employed and evenly distributed among the respondents (Johnson, Lancsar et al. 2013). Each choice card contained three choice alternatives: choices one and two describing scenarios of participating in a clinical trial but differentiated by the levels of the attributes; and choice three was an "opt-out alternative", which gave the respondent the option of not participating in any of the two clinical trial alternatives. For each choice set, respondents were asked to choose their preferred option out of the presented three alternatives. As outlined above, the attributes and corresponding levels were explained via an animated clip briefly before each respondent completed 12 choice sets. Table 2 presents a sample choice set. Along with the choice cards, the survey included supplementary questions on the respondent's understanding of clinical trials, their socioeconomic status, and their health status among other variables. (click here for the link to access the online questionnaire)

\section{Table 2 Sample Choice Set}




\section{Option A Option B Option C}

$\begin{array}{llll}\text { Communication } & \text { GP } & \begin{array}{l}\text { Clinical } \\ \text { trial team }\end{array} & \begin{array}{l}\text { I would prefer not to participate in the } \\ \text { clinical trial. }\end{array}\end{array}$

Likely Benefit No Benefit Moderate Benefit

Risk of side effect High Risk Minimal Risk

Follow up Time

Less than Greater than

one year one year

\section{Please tick the one \\ option you prefer best.}

\section{Results}

The survey was administered online through iReach research company. In total, 535 individuals aged over 18 years, who were already members of the company's online panel, participated in the main study. Individuals were contacted via email by the survey company and invited to take part in the study. Although samples from opt-in panels such as these may be subject to unknown selection biases related to people's unobserved characteristics, the use of this data collection method has grown considerably in recent years due to its relative cost-effectiveness, with no indication of inferior results (Johnston et al., 2017). The sample used in this study represents the population over the age of 18 in Ireland based on gender, age, marital status, education, employment status and attitudes towards care responsibility. Table 3 presents the vital descriptive statistics for the sample. Besides, almost $10 \%$ of respondents reported that they had previously participated in a clinical trial, and $13 \%$ reported that they knew a family member or friend who had participated in a clinical trial. Only $3 \%$ of respondents reporting having worked in clinical trial research, with $6 \%$ of respondents reporting that a family member or friend works in the area of clinical trial research. Respondents were also asked to report how they felt their health was on completing the DCE. Almost two thirds $(70 \%)$ of respondents reported their health to be "very good" or "good". Respondents were asked if they had been diagnosed with a chronic disease. From the list of conditions provided the majority of respondents reported being diagnosed with mental health issues 85 
(16\%), cancer 23(4\%), cardiovascular disease $19(3 \%)$, obesity 40 (7\%), arthritis $57(11 \%)$, diabetes 27 (5\%) disability $17(3 \%)$ and stroke $6(1 \%)$.

\section{Table 3: Descriptive Statistics of the Sample}


Gender

Female

$63 \%(339)$

Male

$37 \%(196)$

Missing

0

Age

$18-24$ |

$6 \%(32)$

$25-34$

$14 \%(78)$

$35-44$

$26 \%(140)$

$45-54$

23\% (122)

$55-64$

$21 \%(111)$

$65-74$

$9 \%$

$75+$

$1 \%$

Missing

0

Education

Primary school education

$2 \%(10)$

Secondary school education

$32 \%$ ( 172)

Third level education

$66 \%(353)$

Missing

0

Relationship status In a relationship

$16 \%(88)$

Married

$53 \%(283)$

Separated/ Divorced/ Widowed

$8 \%(42)$

Single

$23 \%(24)$

Missing

0

Rural/Urban living a city or town in the Republic of Ireland

$41 \%(219)$

a rural part of the Republic of Ireland

$31 \%(166)$

in Dublin city or county

$28 \%(150)$

Missing

0

Page $11 / 26$ 
Both the Medical Card \& Private Health Ins. $\quad 4 \%(23)$

Neither of these

$19 \%(100)$

Missing

0

Employment

Income

Observations
Full-time employment

Home-maker

Part-time employment

Retired

Student

Unemployed

Missing

Less than $€ 12,000$

$€ 12,001$ to under $€ 18,000$

$€ 18,001$ to under $€ 24,000$

$€ 24,001$ to under $€ 30,000$

$€ 30,001$ to under $€ 42,000$

$€ 42,001$ to under $€ 48,000$

$€ 48,001$ to under $€ 60,000$

$€ 60,001$ to under $€ 78,000$

$€ 78,001$ to under $€ 96,000$

$€ 96,001$ or more

Missing
$50 \%(265)$

$12 \%(63)$

$15 \%(81)$

$12 \%(66)$

$5 \%(27)$

$6 \%(33)$

0

$22 \%(119)$

$10 \%(51)$

$10 \%(53)$

$14 \%(74)$

$22 \%(119)$

$7 \%(38)$

$9 \%(48)$

$3 \%(17)$

$1 \%(5)$

$2 \%(11)$

0

535 
The econometric strategy employed for analysing the DCE data was once again informed by the methodological guidance (McFadden and Train 2000, Train 2003). All analysis for the DCE was performed in STATA V.16. The standard econometric framework for the analysis of DCE data is the random utility model (RUM) (McFadden 1986). This model assumes that respondents will choose the alternative that provides the highest level of utility based on the attribute levels of that alternative when presented with several choice alternatives. Attributes vary across choice cards such that the effect of each attribute level on the probability of an alternative being selected can be estimated.

Notably, different discrete choice regression models can be estimated based on the assumptions regarding the error term's distribution. In this paper, we estimate three of the standard regression model specifications employed for DCE analysis. For brevity, we present results from the preferred model in the paper and the present the results from the other two models in an appendix. First, we estimated a conditional logit model, (Louviere, Hensher et al. 2000) which assumes the error term is independent and identically distributed. Second, we estimated a random parameters logit (RPL) model, which accommodates differences in unobserved taste heterogeneity and allows correlation between error terms in each individual's choice, by allowing the attribute coefficient values to differ across individuals (van Osch, Hynes et al. 2017). Third, we estimated a latent class model, in which preferences are assumed to be 'lumpy', such that respondents with similar preferences for the attributes are estimated to belong to the same class. However, respondents who are estimated to have different preferences for the attributes are probabilistically assigned to different classes (Boxall and Adamowicz 2002). The RPL model was the preferred specification, and the results from this analysis are presented. The RPL model is the most commonly used in practice, with the advantages of simplicity, reliability, and easy implementation (Han, Ren et al. 2020).

To allow for an examination of the trade-offs between the attributes, the feature of DCE models, which exploits the monetary attribute employed (if any) and allows for the interpretation of preferences for attributes using a common metric, that is, willingness to accept (WTA) in monetary $€$ value terms. We divide the coefficients for the non-financial compensation attribute levels by the coefficient for the financial incentive attribute to estimate the trade-offs in monetary terms.

\section{Results}

Table 4 below shows findings from the estimated RPL model. In our RPL specification all parameters, except for the financial incentive attribute, are characterised as random parameters with a normal distribution. The model is well specified about the coefficients' statistical significance, indicating that all attributes contributed to respondent preferences. All the parameters of the model are statistically significant at the one percent level.

In terms of the order of importance, the key results are as follows. Respondents stated a strong preference for clinical trials that have a higher likelihood of a benefit for their health and a lower risk of side effects. Respondents indicated their preference for clinical trials with a shorter follow up period. Respondents also indicated a preference for all clinical trial-related information communicated via their 
GP or nurse rather than a clinical trial team. Finally, respondents reported a preference for obtaining a financial incentive to participate in a clinical trial. The alternative regression modelling approaches were generally consistent and confirmative of the RPL findings (see appendix).

In terms of the willingness to accept (WTA) estimates from the RPL model, they may be interpreted as follows. Respondents would need to be compensated $€ 1051$ to participate in a clinical trial with a high risk of side effects relative to a clinical trial with a low risk of side effects. Similarly, respondents would need to be compensated $€ 377$ to participate in a clinical trial with a little or no likelihood of benefit relative to a clinical trial with a high likelihood of benefit. Respondents would need to be compensated $€ 136$ to participate in a clinical trial with a follow up of more than one year. Finally, respondents would need to be compensated $€ 131$ to participate in a clinical trial where communication is handled by the clinical trial team rather than their local doctor or nurse. Notably, these estimates reflect the weight and order of preferences, rather than an actual compensation requirement.

\section{Table 4. Results from the Random Parameter Logit model.}


Communication with the person about the trial

GP

Clinical trial team

Financial Incentive

Likely Benefit

High

Little to none

Moderate

\section{Reference}

$-0.46(.07) \star \star \star \quad 130.78(87.98-173.58)$
$0.003(.0002) * \star \star$

\section{Reference}

$\begin{array}{ll}-1.35(.09)^{\star \star \star} & 377.22(324.89-429.55) \\ -0.50(.07)^{\star \star \star} & 140.70(100.79-180.60)\end{array}$

Risk of side effects

Low

High

Moderate
Reference

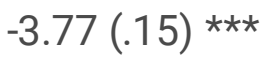

$-1.66(.09) * \star *$
1051.04 (903.51-1198.57)

464.17 (383.28- 545.06)

Follow up time after the trial.

Less than one year

Greater than 1 year
Reference

$-0.48(.07) * \star \star$

$135.73(94.95-176.51)$
ASC

No. of individuals

No. of observations

Log-likelihood

AIC

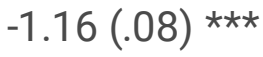

535
$-5049.4194$

12100.07 
Notes: For coefficients, standard errors in parenthesis. For WTA estimates, confidence intervals in parenthesis.

* denotes significant at $10 \%$ level, ** denotes significant at $5 \%$ level and, *** denotes significant at $1 \%$ level.

\section{Discussion}

In this study, we designed and employed a DCE with a representative sample of Irish citizens to explore public preferences for factors that influence an individual's decision to participate in a clinical trial and further, we examine the trade-offs that occur among these factors as part of this decision making process. To the best of our knowledge, this is the first DCE, which has systematically assessed the factors influencing an individual's decision to participate in a clinical trial. The results show that all the five attributes identified through the rigorous pilot phase of the study were proven to be statistically significant factors, influencing the choice of whether or not to participate in a clinical trial, in the econometric data analysis. The findings reveal the magnitude and order of public preferences, for factors such as the likelihood of health benefit, risk of sick effects, follow up, communication and financial incentives. These findings add to the existing evidence base relating to clinical trial participation, offer insight regarding the trade-offs that potential clinical trial participants face in making their decisions, and provide information that may be valuable to those seeking to improve clinical trial recruitment strategies.

Notably, the most important factors or attributes identified in the analysis were those of risk of side effects and likelihood to benefit. These findings are not unsurprising or unexpected, but the empirical results do provide fresh insight into their relative importance. Indeed, a key implication for clinical trial teams may ensure that they clearly articulate these issues in their recruitment approaches. Furthermore, the language used to explain the potential benefits and risks will be a key consideration. This is where the employment of a PPI group can prove so vital and informative. In this study, the PPI group played a crucial role in the design and the questions in the DCE and played particular attention to the concepts of benefit and risk.

Findings from this study indicate that the risk of side effects was identified as an essential factor in the decision-making process as regards clinical trial participation. This is in line with the literature whereby it is reported that perceiving a risk to self was identified as the principal reason why people made a decision not to participate in clinical trials (Walsh and Sheridan 2016). An interesting finding from other studies, which ties into our previous point regarding who communicates information about the trial is that some studies found that patients who were approached by their own doctor and addressed more specifically patients' personal concerns, were more likely to participate to a clinical trial (Greenberg, Corneli et al. 2018). Perhaps the potential disincentive of the perception of risks of side effects can potentially be 
mediated by ensuring patients in the recruitment process are provided with individual attention by health care professionals that are willing and qualified to listen carefully to patient concerns and explain the details to a depth that satisfies the patient. Poor communication between a patient and the medical team can exacerbate patient concerns and reduce opportunities for them to have their concerns addressed. Finally, in line with the literature, we found that altruism and the feeling of contributing to the progress of science were significant incentives for individuals to participate in a clinical trial (Rosenbaum, Wells et al. 2005, Olsen, DePalma et al. 2020). This was captured in the 'likely benefit' attribute, which explicitly referenced 'others', rather than the individual themselves.

Having a friendly and approachable study coordinator is vital as this can not only improve recruitment but also lower the study's dropout rate (Thoma, Farrokhyar et al. 2010). Patients' decisions regarding trial participation may be influenced by the information they receive (Moorcraft, Marriott et al. 2016), and this study further highlights - who delivers that information. This study indicated that participants attached great importance to the relational aspects of the decision-making process, in the focus groups referring to the fact that they trusted their own GP and practice nurse. There is growing evidence that the communication behaviours exhibited by medical and nonmedical professionals tasked with recruiting patients impacts eventual enrolment (Byrne, Kornfeld et al. 2012). Most research on clinical trial communication has focused on general guidelines for communication practice, or how best to communicate trial results to participants (South, Bailey et al. 2019). Strategic communication is an essential element in the practice of recruiting participants in clinical trials. Findings from this study indicate that respondents prefer to have information about the trial communicated to them via their GP or practice nurse as opposed to a clinical trial team member. Notably, healthcare professionals can have a significant impact on recruitment into a clinical trial. Recruitment can suffer when patients perceive support staff to be unavailable or uninterested, or if they have to interface routinely with new staff. Encouraging careful consideration into which healthcare professional communicates initial information about the clinical trial might be expected to lead to better participation.

The results in relation to clinical trial follow up period are once again unsurprising, yet informative. While a number of factors, ranging from informed clinical reasons to budgetary constraints, will influence the choice of length of follow up in a clinical trial, it is clear from our findings that a shorter follow up period should result in higher levels of clinical trial participation.

Findings from the DCE indicate that respondents have a preference to receive a financial incentive with a higher value in order to participate in a clinical trial. Similarly, a recently published pilot study used a DCE to explore and identify preferences for incentives in clinical trials in a population of third-level students and reported that students preferred an incentive with a higher value and compared to no value and preferred cash compared to vouchers (Vellinga, Devine et al. 2020). It is noteworthy. However, earlier studies reported that a more considerable amount offered as an incentive was not found to be more efficacious in motivating a subject to participate (Wertheimer and Miller 2008). As our study highlights, other attributes are essential in the decision-making process regarding clinical trial participation. Some 
studies show that high remuneration is associated with patients' minds as being associated with a perception of higher risk in the trial and thus a reluctance to enrol (Fogel 2018).

Notably, the qualitative research undertaken as part of identifying the DCE attributes highlighted the importance of the financial impact of participating in clinical trials. Focus group participants noted that individuals might have out-of-pocket costs when participating in a clinical trial. These include the cost of transportation and lost work, also medical costs for additional testing. Private Health Insurance may not cover medical care beyond that which is deemed routine. However, we found the use of financial incentives in clinical trials to be not well studied or reported. When consulting with the literature to ascertain the most appropriate value to describe the levels for the financial incentive attribute, we found that whether or not individuals received a financial incentive to take part in a clinical trial, and if so, the amount received was not widely reported. We thus had to consult with clinical trial experts to finalise the levels for this attribute.

This study had a number of limitations, which may go to undermine its findings. Firstly, we examined preference from the publics' perspective and not the patient. There is a debate in the DCE literature as to whose preferences matter and whose preferences ought to be explored -the public or the patient. DCE surveys contain a limited set of testing characteristics; the finite range of attributes and levels is a limitation of DCEs in general. Although the literature review and qualitative research identified a number of potentially important attributes, not all could be considered using this approach. However, by undertaking a systematic approach in selecting the attributes using the nominal group technique, we ensured that the most important were included. Finally, this survey is confined to Ireland, where the findings may not be replicated in other countries. However, given the large nationally representative sample, we believe the findings of the study should be of interest to trialists and clinical trial researchers internationally.

\section{Conclusions}

Given the challenges of clinical trial recruitment, it is of primary importance in designing and executing clinical trials to consider the burden that each individual undergoes during the decision-making process around participation. This study provides an extensive and more in-depth understanding of the individuals reasoning process regarding participation in clinical trials and may, in turn, provide new insights into individuals' views and vulnerability. Although a major motivating factor was the risk of side effects, there was a strong preference for communication of such information to be via respondents GP. We hope that this study will serve as a guide for initial efforts in clinical trial recruitment planning.

\section{Declarations}

Ethics approval and consent to participate Ethical approval for this study was granted by NUI Galway Ethics Committee (application number 18/Sept/18). Informed consent was obtained from all individual participants included in the study. 
Consent for publication All authors and contributors to this research paper consent for the publication of this research article

Availability of data and materials The datasets generated and/or analysed during the current study are available from the corresponding author on reasonable request.

Competing interests Michelle Queally declares no conflicts of interest. Declan Devane declares no conflicts of interest. Matthew Griffin declares no conflicts of interest. Abhay Pandit is the Director of CÚRAM, SFI Research Centre for Medical Devices. Paddy Gillespie declares no conflicts of interest.

Funding This research has emanated from research conducted with the financial support of Science Foundation Ireland (SFI) and is co-funded under the European Regional Development Fund under Grant Number 13/RC/2073_P2.

Author contributions All authors contributed to the development of the concept and design of the study. MQ undertook the design of the DCE, the statistical analysis and prepared a first draft of the manuscript. $P G, D D$ and $M G$ contributed to the design of the discrete choice experiment in particular the attributes used. All authors contributed to the interpretation and writing the manuscript. PG and AP obtained funding for data collection. All authors played a role in the review of the analysis, interpretation of the results.

Acknowledgements We would like to thank all members of the public who participated in the focus groups. We would also like to thank all member of the Public Patient Involvement Group and member of the PPI Ignite team at NUI Galway who helped inform this study.

\section{References}

Bech, M., et al. (2011). "Does the number of choice sets matter? Results from a web survey applying a discrete choice experiment." Health Economics 20(3): 273-286.

Bliemer, M. C., et al. (2008). "Approximation of Bayesian efficiency in experimental choice designs." Journal of Choice Modelling 1(1): 98-127.

Boxall, P. C. and W. L. Adamowicz (2002). "Understanding heterogeneous preferences in random utility models: a latent class approach." Environmental and Resource Economics 23(4): 421-446.

Byrne, M. M., et al. (2012). "Discussions of cancer clinical trials with the National Cancer Institute's Cancer Information Service." Journal of Health Communication 17(3): 319-337.

Carlisle, B., et al. (2015). "Unsuccessful trial accrual and human subjects protections: an empirical analysis of recently closed trials." Clinical Trials 12(1): 77-83.

Chu, S. H., et al. (2015). "Factors associated with willingness to participate in clinical trials: a nationwide survey study." BMC Public Health 15(1): 10. 
Coyle, M. and K. Gillies (2020). "A systematic review of risk communication in clinical trials: How does it influence decisions to participate and what are the best methods to improve understanding in a trial context?" PLoS One 15(11): e0242239.

Fogel, D. B. (2018). "Factors associated with clinical trials that fail and opportunities for improving the likelihood of success: a review." Contemporary Clinical Trials Communications 11: 156-164.

Greenberg, R. G., et al. (2018). "Perceived barriers to pediatrician and family practitioner participation in pediatric clinical trials: Findings from the Clinical Trials Transformation Initiative." Contemporary Clinical Trials Communications 9: 7-12.

Gul, R. B. and P. A. Ali (2010). "Clinical trials: the challenge of recruitment and retention of participants." Journal of Clinical Nursing 19(1-2): 227-233.

Han, B., et al. (2020). "Mixed Logit Model Based on Improved Nonlinear Utility Functions: A Market Shares Solution Method of Different Railway Traffic Modes." Sustainability. 12(4): 1406.

Harrison, M., et al. (2014). "Risk as an attribute in discrete choice experiments: A systematic review of the literature." The Patient-Patient-Centered Outcomes Research 7(2): 151-170.

Healy, P., et al. (2018). "Identifying trial recruitment uncertainties using a James Lind Alliance priority setting partnership-the PRioRiTy (Prioritising recruitment in randomised trials) study." Trials 19(1): 147.

Horton, J. (1980). "Nominal group technique: A method of decision-making by committee." Anaesthesia 35(8): 811-814.

Johnson, F. R., et al. (2013). "Constructing experimental designs for discrete-choice experiments: report of the ISPOR conjoint analysis experimental design good research practices task force." Value in Health 16(1): 3-13.

Kerr, D. J., et al. (2008). Clinical trials explained: A guide to clinical trials in the NHS for healthcare professionals, John Wiley \& Sons.

Kurt, A., et al. (2017). "Factors influencing participation in clinical trials: emergency medicine vs other specialities." Western Journal of Emergency Medicine 18(5): 846.

Kurt, A., et al. (2017). "Racial differences among factors associated with participation in clinical research trials." Journal of Racial and Ethnic Health Disparities 4(5): 827-836.

Lancaster, K. J. (1966). "A new approach to consumer theory." The Journal of Political Economy: 132-157.

Lancsar, E. and J. Louviere (2008). "Conducting discrete choice experiments to inform healthcare decision making." Pharmacoeconomics 26(8): 661-677. 
Lancsar, E. and J. Louviere (2008). "Conducting Discrete Choice Experiments to Inform Healthcare Decision Making: A Users Guide." Pharmacoeconomics 26(8): 661-677.

Louviere, J. J., et al. (2000). Stated choice methods: analysis and applications, Cambridge University Press.

McCann, S. K., et al. (2010). "Reasons for participating in randomised controlled trials: conditional altruism and considerations for self." Trials 11(1): 31.

McFadden, D. (1986). "The choice theory approach to market research." Marketing Science 5(4): 275-297.

McFadden, D. and K. Train (2000). "Mixed MNL models for discrete response." Journal of Applied Econometrics 15(5): 447-470.

Moorcraft, S. Y., et al. (2016). "Patients' willingness to participate in clinical trials and their views on aspects of cancer research: results of a prospective patient survey." Trials 17(1): 1-12.

Newton, S. K. and J. APPIAH-POKU (2007). "Opinions of researchers based in the UK on recruiting subjects from developing countries into randomised controlled trials." Developing World Bioethics 7(3): 149-156.

Olsen, L., et al. (2020). "Self-Interested and Altruistic Motivations in Volunteering for Clinical Trials: A More Complex Relationship." Journal of Empirical Research on Human Research Ethics: 1556264620914463.

Pearce, A., et al. (2020). "Respondent Understanding in Discrete Choice Experiments: A Scoping Review." The Patient-Patient-Centered Outcomes Research: 1-37.

Rosenbaum, J. R., et al. (2005). "Altruism as a reason for participation in clinical trials was independently associated with adherence." Journal of Clinical Epidemiology. 58(11): 1109-1114.

Ryan, M. (1999). "A role for conjoint analysis in technology assessment in health care?" International Journal of Technology Assessment in Health Care 15(03): 443-457.

Ryan, M., et al. (2007). Using discrete choice experiments to value health and health care, Springer Science \& Business Media.

Schilling, l., et al. (2019). "Patient involvement in clinical trials: motivation and expectations differ between patients and researchers involved in a trial on urinary tract infections." Research Involvement and Engaggement 5(1): 15.

Sood, A., et al. (2009). Patients' attitudes and preferences about participation and recruitment strategies in clinical trials. Mayo Clinic Proceedings, Elsevier. 
South, A., et al. (2019). "Effectiveness and acceptability of methods of communicating the results of clinical research to lay and professional audiences: protocol for a systematic review." Systematic Reviews 8(1): 150.

Spinks, J. and D. Mortimer (2015). "Lost in the crowd? Using eye-tracking to investigate the effect of complexity on attribute non-attendance in discrete choice experiments." BMC Medical Informatics and Decision Making 16(1): 14.

Thoma, A., et al. (2010). "How to optimise patient recruitment." Canadian Journal of Surgery 53(3): 205.

Train, K. (2003). Discrete choice methods with simulation, Cambridge university press.

Treweek, S., et al. (2018). "Strategies to improve recruitment to randomised trials." $\underline{\text { Cochrane Database of }}$ Systematic Reviews(2).

van Osch, S., et al. (2017). "Estimating the Irish public's willingness to pay for more sustainable salmon produced by integrated multi-trophic aquaculture." Marine Policy 84: 220-227.

Vellinga, A., et al. (2020). "What do patients value as incentives for participation in clinical trials? A pilot discrete choice experiment." Research Ethics 16(1-2): 1-12.

Walsh, E. and A. Sheridan (2016). "Factors affecting patient participation in clinical trials in Ireland: a narrative review." Contemporary Clinical Trials Communications 3: 23-31.

Wertheimer, A. and F. G. Miller (2008). "Payment for research participation: a coercive offer?" Journal of Medical Ethics 34(5): 389-392.

\section{Appendix}

Table 5. Results from the conditional logit model. 
Communication with the person about the trial

GP

Clinical trial team
Reference

$-0.19(.04) * \star \star$

$83.29(46.37-120.21)$

Financial Incentive

Likely Benefit

High

Little to none

Moderate
Reference

$-0.88(.05) * \star \star$ $350.89(297.52$ - 404.26$)$

$-0.30(.04) * \star \star$ $130.46(89.27-171.65)$

\section{Risk of side effects}

Low

High

Moderate
Reference

$-1.99(.05)$ *** $860.91(732.32-989.50)$

$-1.02(.06) * \star \star \quad 441.92(362.74-521.10)$

Follow up time after trial.

Less than one year

Greater than one year

ASC-opt-out
Reference

$-0.24(.04) * * *$

$104.30(68.77-139.82)$

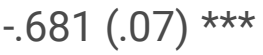

No. of individuals

No. of observations

Log-likelihood

AIC

BIC

\section{5}

19,260

$-6042.0328$

10006.63

10116.75 
Notes: For coefficients, standard errors in parenthesis. For WTA estimates, confidence intervals in parenthesis.

* denotes significant at $10 \%$ level, $* \star$ denotes significant at $5 \%$ level and, $* \star \star$ denotes significant at $1 \%$ level.

Table 6. Results from the latent class model. 
Communication with a person about the trial

GP Reference Reference Reference

Clinical trial team

\begin{tabular}{llll} 
& -0.35 & 0.086 & -0.14 \\
& $(.12) \star \star \star$ & $(.73)$ & $(.04) \star \star \star$ \\
\hline Financial Incentive & 0.005 & 0.002 & 0.003 \\
& $(.0004) \star \star \star$ & $(.002)$ & $(.0004) \star \star \star$
\end{tabular}

Benefit (as a result of participating in the clinical trial)

High

Little to none

Moderate

Reference Reference Reference

$\begin{array}{lll}-1.81 & -1.39 & -0.87 \\ (.21) \star \star \star & (.67) * \star & (.07) \star \star \star \\ -0.90 & -1.29 & -0.21 \\ (.13) \star \star \star & (.53) \star * & (.06) \star \star \star\end{array}$

Risk of side effects (as a result of participating in the clinical trial)

Low

High

Moderate

Reference Reference Reference

$\begin{array}{lll}-5.27 & -2.30 & -1.42 \\ (.30) \star \star \star & (.60) * \star * & (.07) \star \star \star \\ -2.84 & -2.03 & -0.47 \\ (.14)^{\star \star \star} & (.62)^{\star \star \star} & (.07) \star \star \star\end{array}$

Follow up time after trial.

Less than one year

Greater than one year
Reference

$-0.70$

$(.11) * \star \star$
Reference Reference

$-0.97 \quad-0.05$

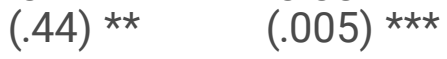
Ascd 
Notes: For coefficients, standard errors in parenthesis.

* denotes significant at $10 \%$ level, $* *$ denotes significant at $5 \%$ level and, $* * \star$ denotes significant at $1 \%$ level.

\section{Figures}

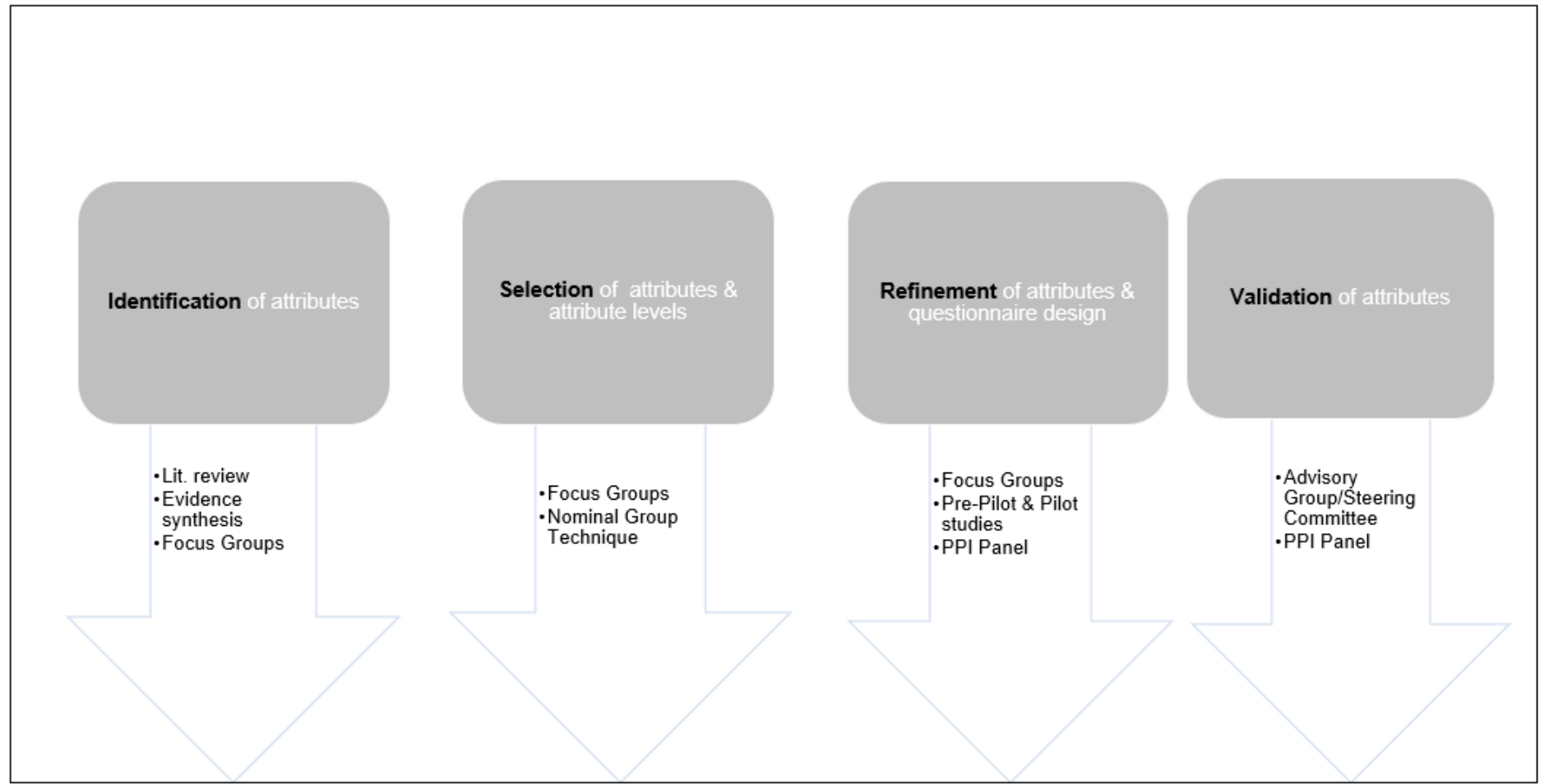

\section{Figure 1}

Attribute Development Pathway

\section{Supplementary Files}

This is a list of supplementary files associated with this preprint. Click to download.

- EquatorNetworkReportingChecklist.docx

- SuggestedReviewers.docx 